\title{
BEND OVER SHEAVE DURABILITY OF FIBRE ROPES FOR DEEP SEA HANDLING OPERATIONS
}

\author{
P. Davies, N. Lacotte \\ IFREMER \\ Brest, France
}

\author{
S. François, O. Lodeho \\ Subsea7 \\ Paris, France
}

\author{
D. Durville, D. Vu \\ Ecole Centrale de Paris \\ Paris, France
}

\author{
G. Kibsgaard \\ Technip \\ Paris, France
}

\author{
K. Konate, S. Mills \\ IMECA \\ La Rochelle, France
}

\author{
R. Craig, D. Cannell \\ Technip \\ Aberdeen, Scotland
}

\author{
M. François, A-L Defoy \\ Bureau Veritas \\ Paris, France
}

\begin{abstract}
This paper describes work performed within a Joint Industry Project aiming to evaluate the lifetime of deep sea handling ropes. Various HMPE (High Modulus Polyethylene) fiber ropes, with and without coatings, have been studied under both tensile and cyclic bend over sheave (CBOS) loading. A large test program has enabled both tension-cycle to failure relationships and empirical expressions for residual strength after cycling to be determined. A special device was then developed to apply a known couple to the sheave, allowing both dynamic friction measurements to be made and the influence of applied couple on cycles to failure to be measured. These experimental data were used in the development of a numerical model which can be used to study the influence of rope and sheave parameters.
\end{abstract}

\section{INTRODUCTION}

There has been considerable work to examine how steel wire ropes behave when they are used in handling operations and pass repeatedly over sheaves. This is a key issue in the design of many lifting applications, from building elevators to mining and various authors have presented test results $(1,2)$.

Concerning large offshore steel wire handling ropes Vennemann et al. presented some results at a previous OMAE conference (3). When operations must be performed in deep sea steel wire becomes less efficient, supporting its own weight rather than payload, and synthetic fiber ropes become very attractive. One example is the use of both aramid and HMPE ropes for deep sea oceanography operations such as coring, at depths down to 6000 meters (4). The offshore oil and gas industry is extending its requirements to depths in excess of 2000 meters and is also becoming very interested in synthetic ropes. Bend over sheave performance is one of the aspects which are of interest for ropes designed to install sub-sea equipment weighing 200 tons or more at these depths. However, more generally it is essential for CAPEX (Capital Expenditure) requirements to know how long such ropes will last, and whether existing winch equipment can handle them. Unfortunately few data are available to help the end user to answer these questions. The DISH JIP's collected some information (5) but this is still mostly confidential. There are other projects underway, e.g. SIRIUS, "Safe Installation with Ropes in Ultradeep Sea" (6) and ODIM (7) which are generating some large scale CBOS data for the offshore industry, but again the test details and results are mostly confidential at present.

Synthetic ropes offer the possibility to design a product specifically for the application. For example, external friction between the rope and the winch should be high, as this will determine the winch size, and this can be achieved by an external coating. On the other hand internal friction should be 
low to minimize wear and extend lifetime, and various strategies have been proposed to enhance this (8).

In this paper a JIP involving offshore engineering companies (Technip, Subsea7), a winch maker (IMECA), a Classification Society (BV), a rope supplier (Samson Ropes), an engineering school (Ecole Centrale de Paris) and the French Ocean Research Institute (IFREMER) will be described. This project, which started in 2010, has been addressing long term durability of deep sea handling ropes.

First, the test equipment and materials will be described. The tensile properties of these materials will be given, then some examples of CBOS and residual strength results will be discussed. Numerical model development will then be described, and some model results will be given. Finally damage development in HMPE ropes under cyclic flexural loading on sheaves will be discussed.

\section{TEST EQUIPMENT}

Assembled yarn samples were tested on a $10 \mathrm{kN}$ tensile test machine. The main originality of this equipment is the pneumatic grips, developed for testing yarn samples, and the two digital cameras used to follow the movement of two markers attached to the yarn. These provide non-contact extensometry when analyzed using in-house image analysis software. Figure 1 shows the test set-up.

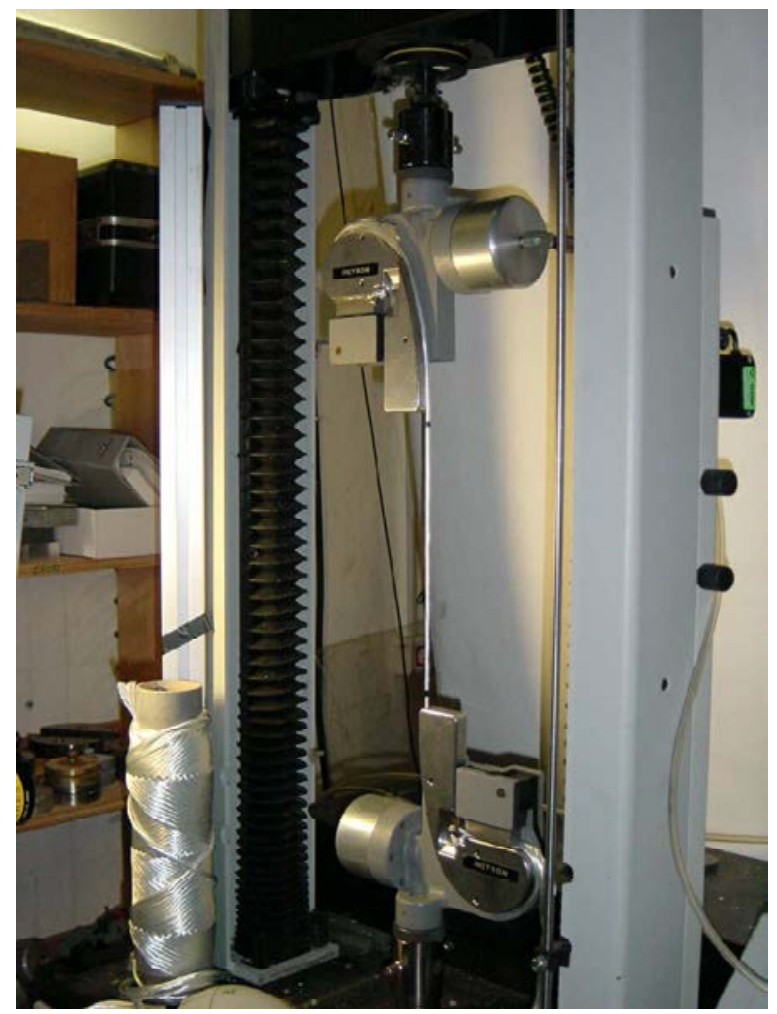

Figure 1. Tensile test set-up
The rope samples were all tested on two machines at the Ifremer centre in Brest. The first is a 100 ton tensile test frame, Figure 2. This is also equipped with the non-contact video extensometry system, mounted on a rail above the machine.

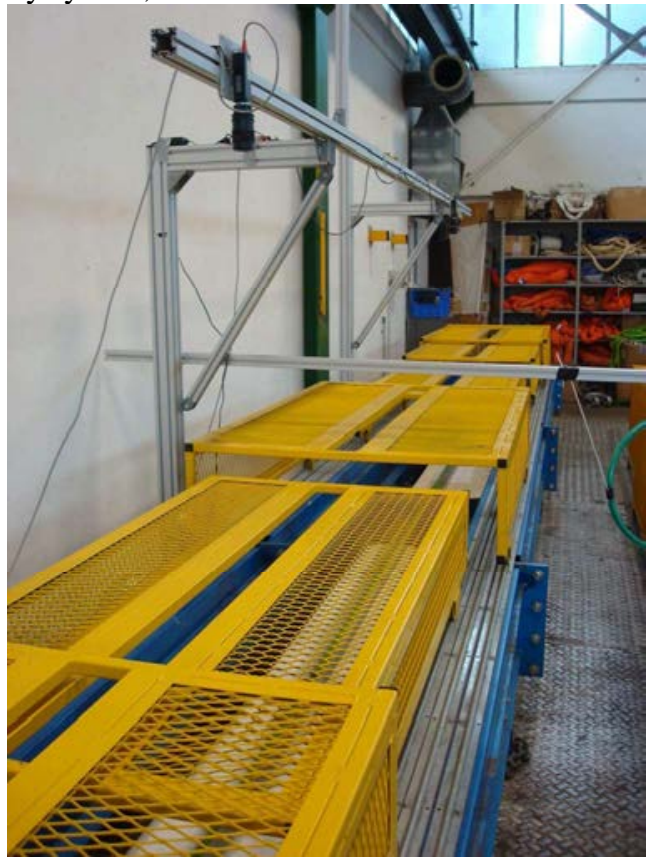

Figure 2. $1000 \mathrm{kN}$ tensile test frame, showing video extensometer system mounted above machine.

The second is a specially designed cyclic bend over sheave (CBOS) test frame, Figure 3, which allows ropes to be loaded up to $250 \mathrm{kN}$ by a central hydraulic ram and cycled over a sheave of diameter up to 1.5 meters by two external rams, each with a stroke of 3 meters. Loads and displacements of all three rams are measured continuously throughout the tests. Water pumps allow the samples to be wetted continuously during tests if required.

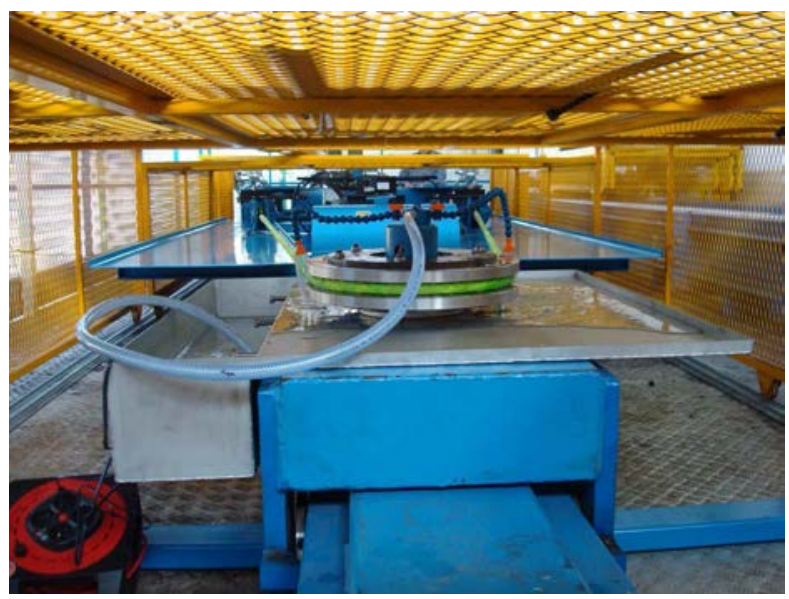

Figure 3. CBOS test machine, showing water spray device. 
This CBOS machine was adapted by IMECA to enable a torque to be applied to the sheave during cycling, as shown in Figure 4. Additional measurements can then be made of both torque and motor speed, the latter allowing the onset of slipping to be clearly identified.

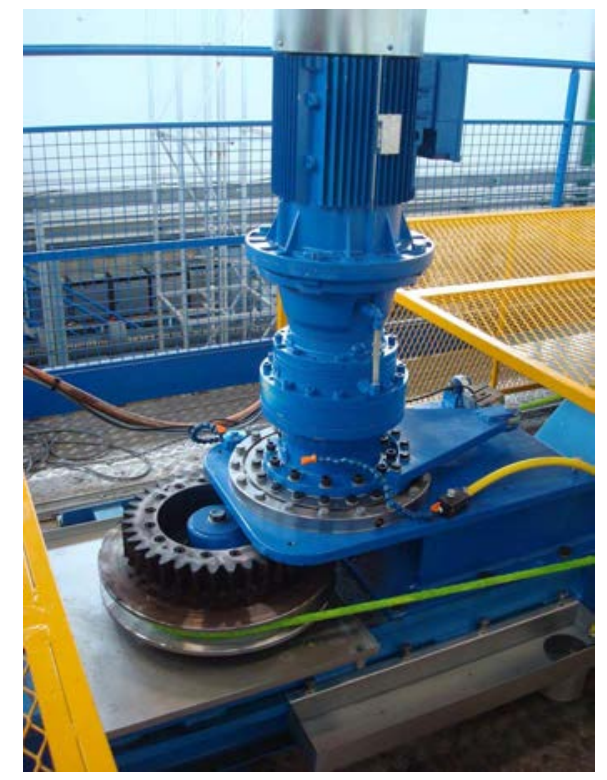

Figure 4. Device allowing known torque to be applied to sheave

In order to examine both the details of the rope construction and the development of damage during cycling, $\mathrm{X}$ ray micro-tomography has been used. This technique involves rotating a sample in an X-ray beam and recording the images which can then be used to reconstruct a 3-D image of the interior of the sample. Figure 5 shows the equipment used.

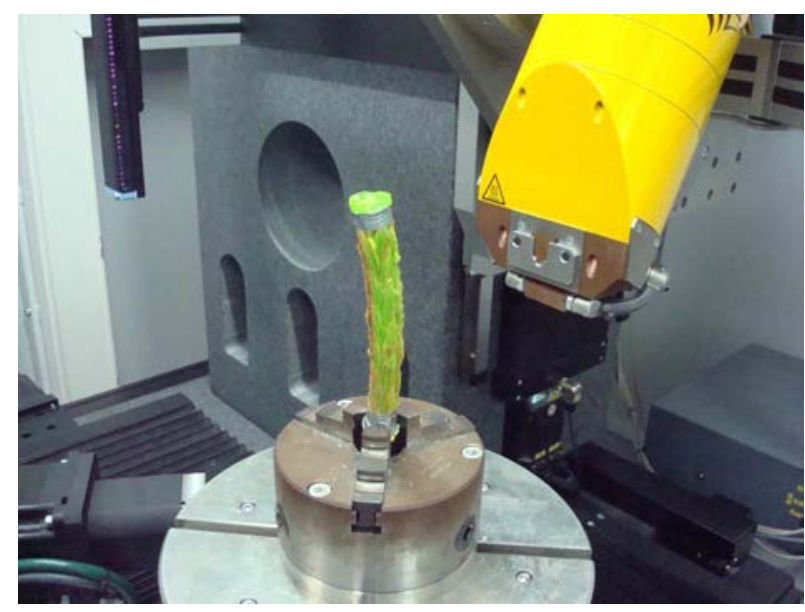

Figure 5. CBOS damaged rope sample section during examination in $\mathrm{X}$ ray tomography chamber.

\section{MATERIALS}

The materials tested were all braided ropes provided by Samson Ropes and all are based on HMPE fibres. There is a reference material (Amsteel Blue) and three modified versions, including samples based on DPX patented processing technology [9]. Figure 6 shows examples of load-strain plots for tensile tests on assembled yarns which had been removed from the reference material rope.

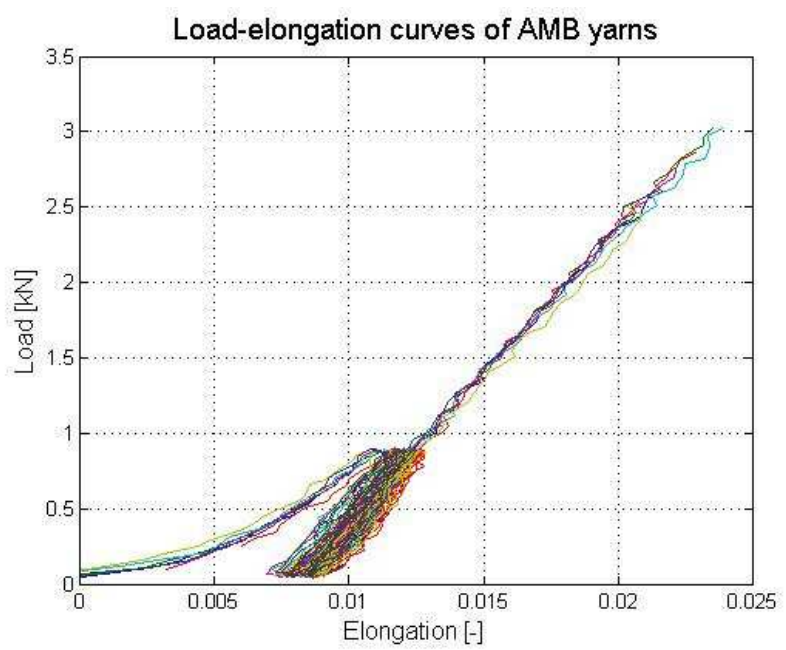

Figure 6. Yarn tensile test plots (5 samples)

Figure 7 shows an example of a tomography image of the rope structure under low load.

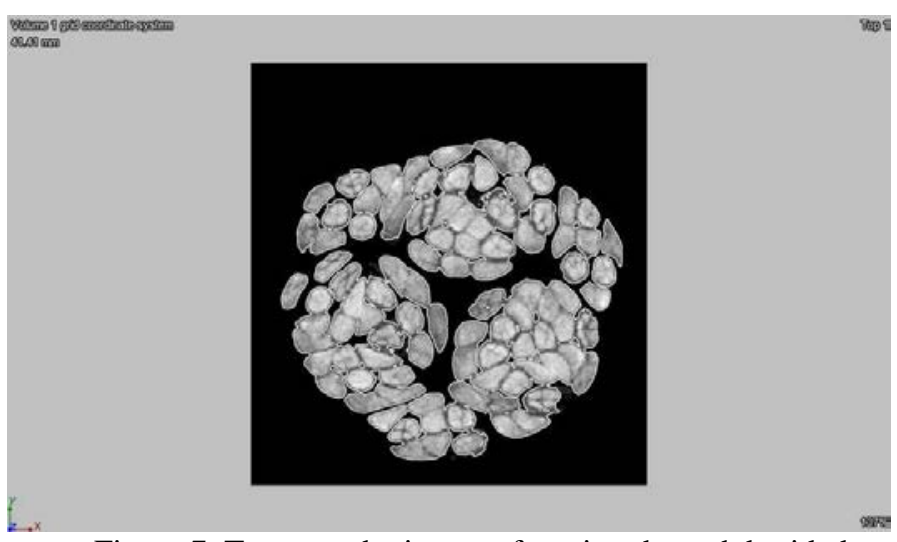

Figure 7. Tomography image of section through braided rope sample before testing.

These show the complexity of the rope structure. This is a 12-strand braid but the assembled yarns in each strand are far from circular, and their shape changes as we move along the rope. 


\section{EXAMPLES OF TEST RESULTS}

Tensile tests were performed on spliced rope samples, both to characterize the materials and to provide results to compare with modeling predictions. Figure 8 shows an example of two break tests on a braided sample; for one test direct ramping to failure was applied, while for the second the rope was cycled before the failure ramp. These illustrate the well-known bedding-in phenomenon, caused by molecular realignment, construction re-orientation, and load redistribution within the rope. After bedding-in the rope response is almost linear under these conditions.

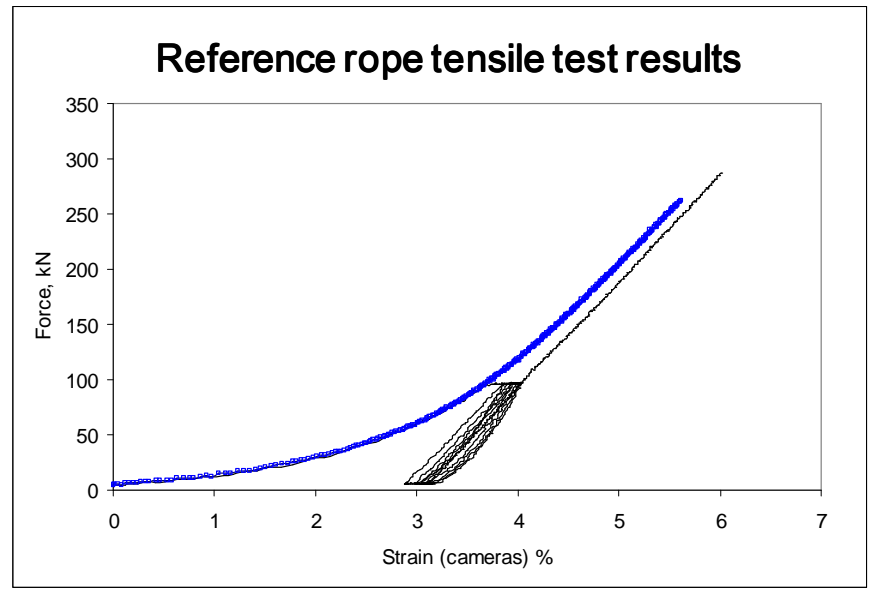

Figure 8. Example of tensile test plots, reference HMPE. Blue curve is continuous test to fail, black curve includes 10 load-unload cycles to $100 \mathrm{kN}$ before ramp to fail.

Rope samples were tested to failure on sheaves with a D/d ratio (sheave diameter/rope diameter) of 20. Various sheave profiles were examined. The period was 20 seconds for a complete back and forward cycle of the rope onto the sheave and back off.

A first series of tests was performed to examine the influence of the technique used to measure residual strength on safety factors. This is a key issue for a test program, so both straight tensile pull and cycling to failure on the sheave at increasing load levels were examined. An example of the results is shown in Figure 9. Residual strength is plotted versus the number of cycles $\mathrm{N}$ normalized by the cycles to failure $\mathrm{N}_{\mathrm{f}}$.

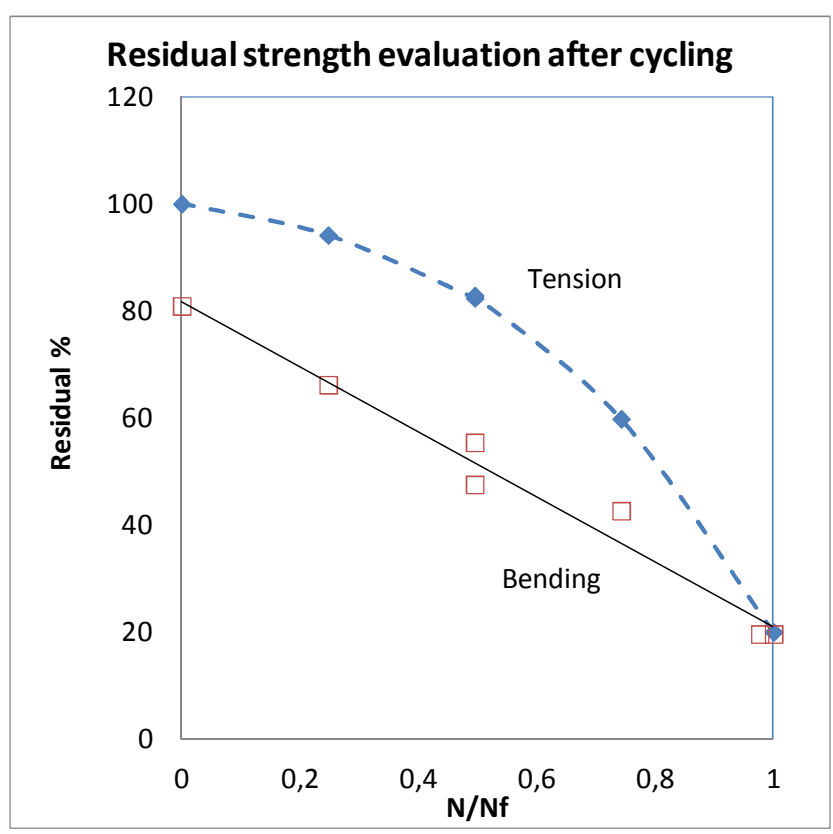

Figure 9. Residual strengths (\% of initial tensile strength) measured after cycling to different percentages of lifetime at $20 \%$ break load. Residual values evaluated by either tensile direct pull (filled diamond symbols) or by cycling on sheave at increasing load (open squares).

These results are very important as they clearly show that it is dangerous to rely on residual tensile strengths to evaluate the influence of cyclic bending damage, as they will provide an over-optimistic value of residual strength. Cyclic bending on the sheave at increasing load was therefore used in all subsequent tests to evaluate remaining lifetime.

The determination of data such as those in Figure 9 can then be used in an empirical life prediction model. For this it is essential to run additional tests with more complex loading sequences, in order to validate the predictions. Such tests are underway.

Both wet $\left(20^{\circ} \mathrm{C}\right.$ water temperature $)$ and dry $\left(20^{\circ} \mathrm{C}\right.$ air temperature) tests were performed initially but the majority of tests were performed with continuous wetting. Rope surface temperatures were measured using an IR camera, which proved particularly useful when couples were applied to the sheave. In this case slipping could occur and large temperature rises $\left(>30^{\circ} \mathrm{C}\right)$ were noted. In the majority of tests however, temperature rises remained below $10^{\circ} \mathrm{C}$, Figure 10 . 


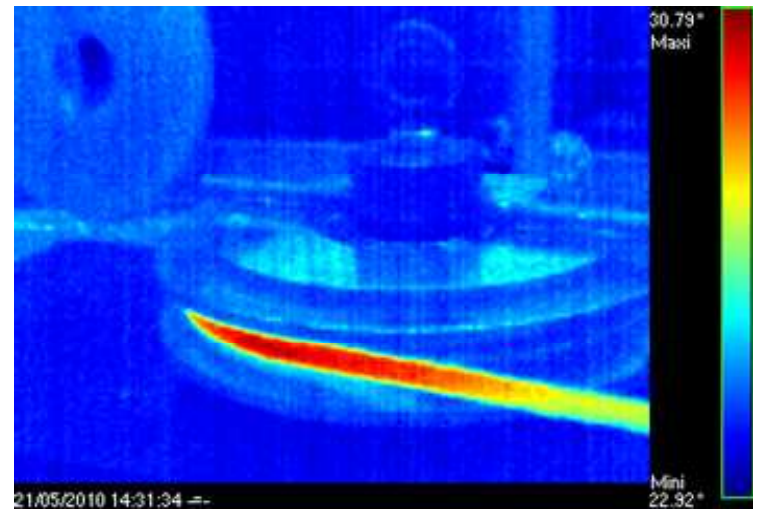

Figure 10. Example of IR camera image during CBOS test

By modifying the fibre coating it is possible to obtain significantly improved test results. This is illustrated in Figure 11, where tension versus cycles-to-failure plots for a standard reference HMPE and an improved version are compared. The cycles to failure are normalized with respect to values at the same load level for the reference material, and improvements of $5 \mathrm{x}$ to $7 \mathrm{x}$ are measured.

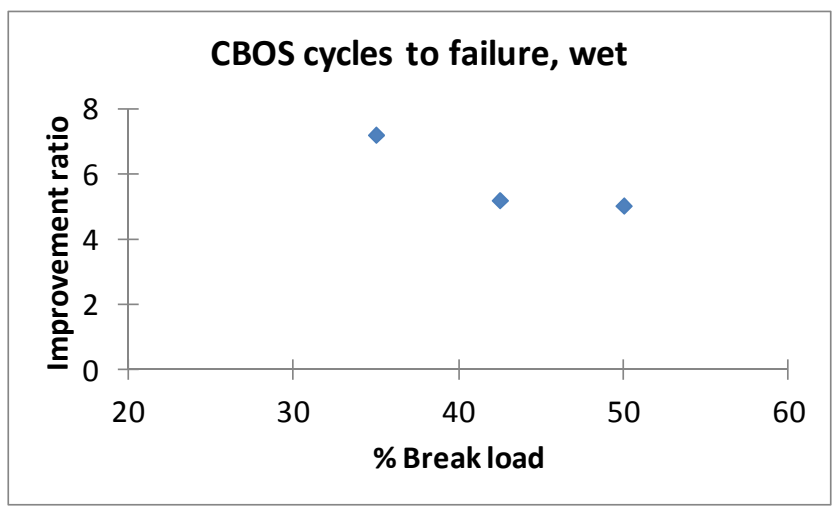

Figure 11. Improvement in CBOS performance due to coating, ratio between cycles to failure for improved coating samples and cycles for reference HMPE at the same load levels.

While these results show that CBOS behavior can be significantly improved, these ropes, in addition to passing over sheaves must also be wound onto a winch. The low internal friction resulting from the coatings required for improved CBOS behavior are then a disadvantage as they also reduce the external sheave to rope friction. In order to maintain CBOS performance and improve external friction more sophisticated coatings are required. Their effectiveness can be quantified by testing them on the equipment shown in Figure 4.

A number of materials have been tested on this device in order to examine their external friction behavior under cyclic loading conditions at different load levels, both wet and dry. It is very important to determine how these materials behave throughout their lifetime, not just initially, as the rope evolves with time. Figure 12 shows one example of a result for both the reference HMPE (no coating) and an improved friction coating.

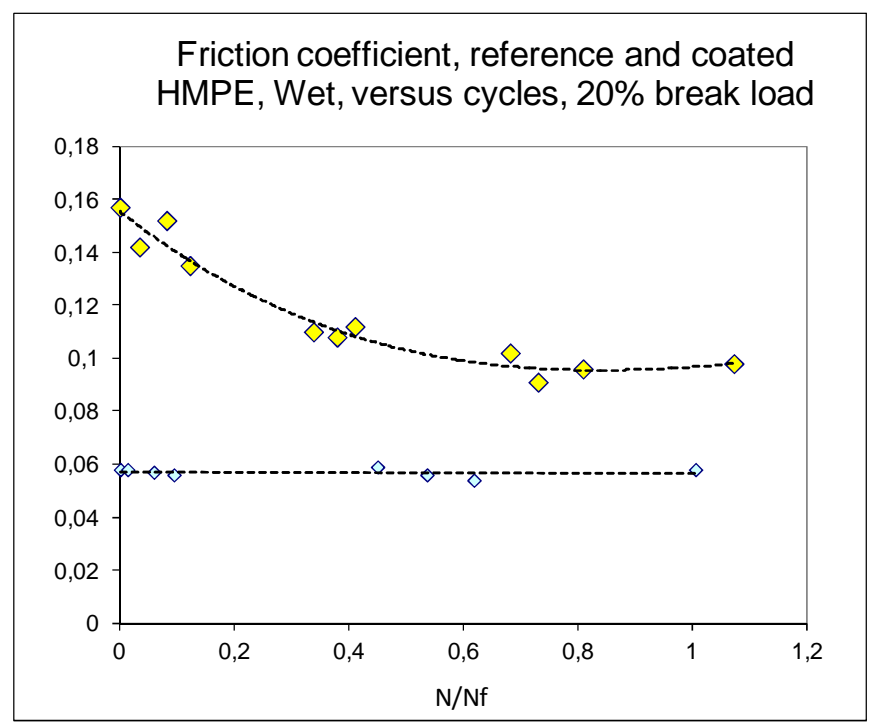

Figure 12. Example of sheave/rope friction coefficients versus cycles (N/Nf) for reference (blue symbols) and modified ropes (yellow symbols).

Friction coefficients were determined by cycling and progressively increasing the applied couple until slipping occurred. The coefficient $\mu$ was determined from the measured loads (T) in the two outer rams at slippage and the contact angle $\theta$, using the expression: $T_{\text {high }} / T_{\text {low }}=e^{\mu \theta}$.

In this case the friction coefficient of the modified material is clearly significantly higher than that of the reference rope, even at the end of its lifetime.

\section{NUMERICAL MODELLING}

In parallel with the empirical lifetime prediction modeling described above, a numerical rope model was also developed. The main objective of this development was to create a tool which could:

- Enable parametric studies to be performed, so that rope and sheave properties could be optimized, and

- To study scale changes in order to establish the extent to which small rope data could be applied to larger ropes.

The model employed here was a specially developed application of the Multifil software (10-12). One of the particular features of this finite element software is that it allows a large number of contact points to be analyzed rapidly.

Two aspects of contact are known:

- The contact takes place in a fixed zone

- The direction of the research: symmetrical

There are however some special points in a fibrous milieu: The contact can appear anywhere, and the normal contact direction 
is asymmetrical, which implies an interaction of the calculation of the normal directions between the rope elements. To overcome these obstacles, two solutions are proposed in Multifil: Proximity zones and intermediate geometry. To locate the zone in which contact between two rope elements (fibers or yarns) occurs, a coarse mesh is created along the fiber on the curved abcissa. The mesh step is pre-defined. For each node the minimal distance to another fiber is obtained by projecting perpendicularly to that fiber. The nodes for which the minimal distance is less than the initial research distance (a few times the fiber radius) are grouped in a zone termed "proximity zone". A pair of rope elements can have several proximity zones. The creation of the intermediate geometry is the mean line in the proximity zone. The contact appears when the surfaces of two elements approach this intermediate geometry.

The first step was to simulate the geometry of the braided samples. In the simulation, for the starting geometry we introduce the trajectory of each assembled yarn and the radius of the transverse section, together with the distribution of the assembled yarns and the strands in the plane perpendicular to the braid axis. The initial length of the braid is also entered. Tensile testing of assembled yarns provides input stiffness data. Figure 13 shows the geometric model, Figure 14 shows the comparison between model and tensile test results.

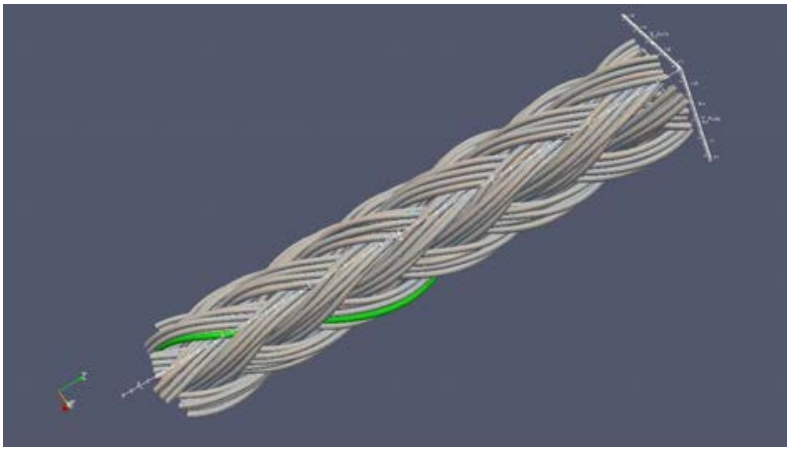

Figure 13. Braided rope model, one lay length

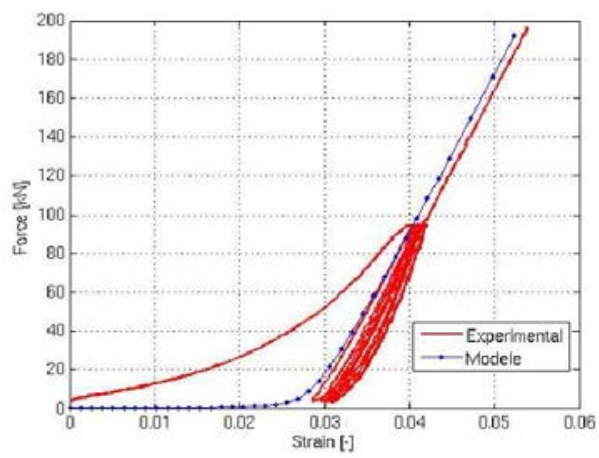

Figure 14. Experimental and model results for tensile break test on braided HMPE rope.
The braided elements are pulled together in the model until contact occurs and the load starts to increase.

The next step was to simulate the bending of this rope over a sheave. Figure 15 shows an example of a simulation, intended to reproduce the experimental test set-up.

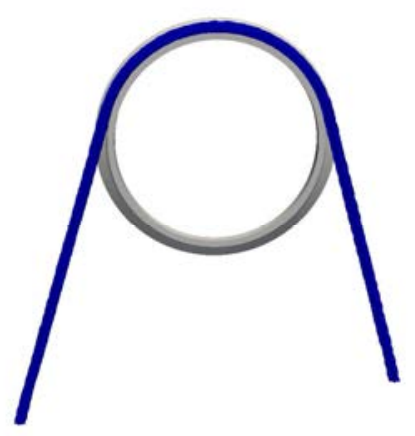

Figure 15. Bend over sheave simulation geometry

Figure 16 shows a detail of the rope construction and the sheave profile.

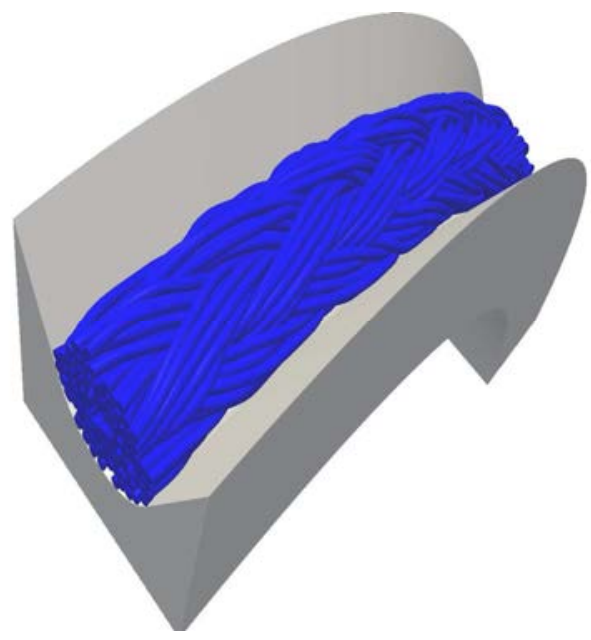

Figure 16. Detail of rope bending model.

The third step was to model the cycling of the rope back and forwards over the sheave. Various cases have been studied and one example of results is shown below, Figure 17. This illustrates how the sensitivity of the loading to various input values can be examined. Here, the energy dissipated by internal and external friction contacts has been quantified for one set of friction coefficients during the first loading cycles. 


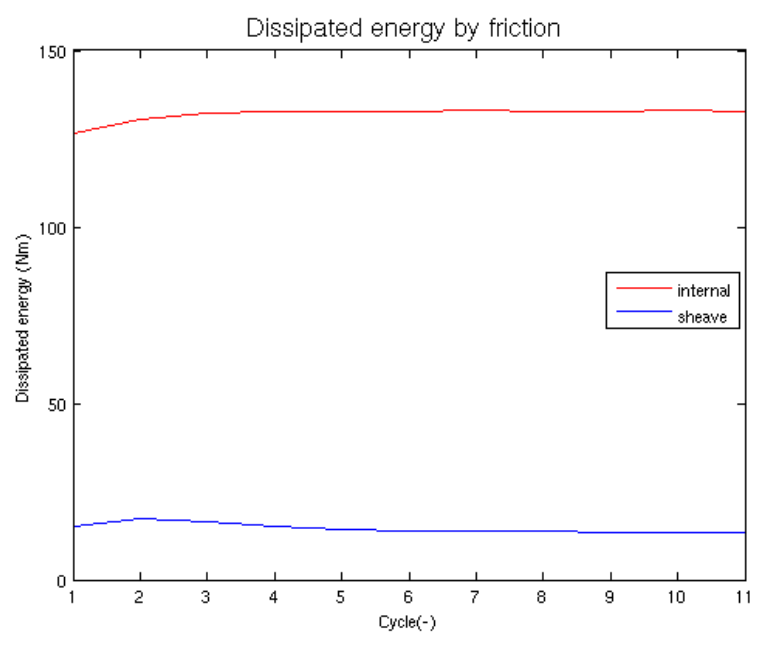

Figure 17. Example of results for dissipated energy during cycling at $20 \%$ break load, friction coefficients:

0.03 internal, 0.10 external.

This shows that under these conditions it is the internal abrasion rather than the external pulley/sheave interactions which will govern the rope lifetime. Such information is valuable in designing an optimal rope for a given operation.

\section{DAMAGE DEVELOPMENT DURING CYCLIC LOADING}

In order to extend the model to include changes resulting from damage development, the different damage mechanisms and their development during cycling must be understood. This is a complex task due not only to the many geometrical interactions, both between the individual rope elements and between the rope and the sheave, but also to the possibility of coupling between rope properties and temperature changes. Various techniques have been applied to examine rope damage, including interrupting tests at various stages and examination. Microscopy studies provide a first indication of damage processes; Figure 18 shows two aspects, the smooth inner contact surface with the sheave, where local melting has occurred, and the extruded fibers on the outer surface which reduce the load-carrying capacity of the rope.

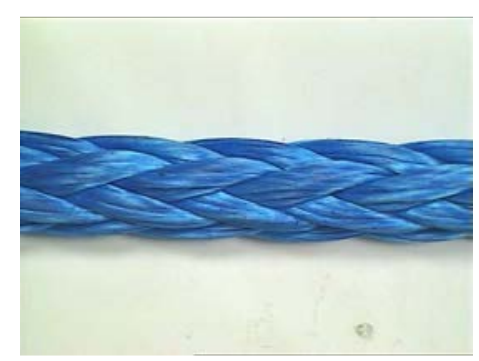

(a)

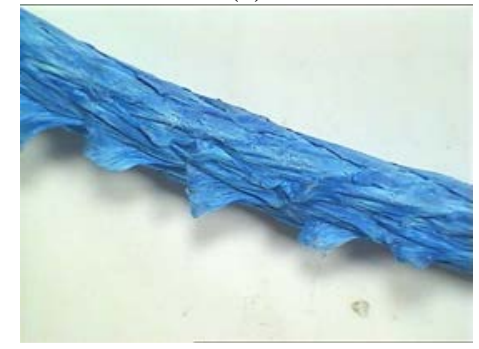

(b)

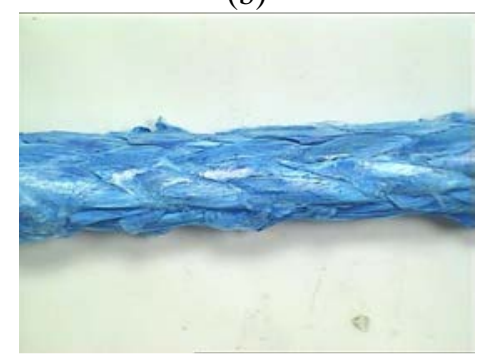

(c)

Figure 18. Optical microscopy images of HMPE rope a) New

b) External surface damage, interrupted CBOS test

c) Smooth internal surface (in contact with sheave), interrupted CBOS test.

Such visual observations provide some indications of surface degradation, but X-ray micro-tomography is a powerful complementary tool which can provide much additional information on what is happening inside the rope. Figure 19 shows an example of images from an interrupted test. Permanent changes to the shape of the rope and its internal structure can be quantified, together with detailed evaluation of yarn damage. 


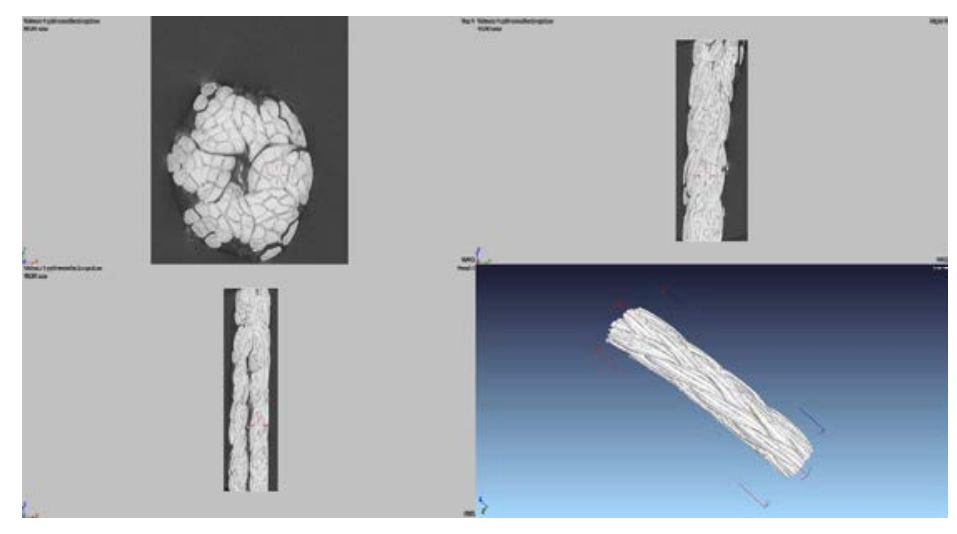

Figure 19. One example of reconstructed X-ray microtomography images of rope sample, interrupted CBOS test.

\section{CONCLUSIONS}

This paper provides some examples of results from a Joint Industry Project examining durability of deep sea handling ropes. The project has generated a data base of experimental results on various HMPE fiber ropes with and without coatings. Tensile tests, CBOS and dynamic friction measurements have been performed, and are now being used to develop empirical life prediction models. In parallel, a numerical model has been developed which allows parametric studies to be performed, and this will be employed to investigate scale changes as rope size is increased. X-ray micro-tomography images are being used to relate rope damage during cycling to modeling predictions.

\section{REFERENCES}

(1) Feyrer K, Wire ropes, Tension, Endurance, Reliability, Springer Berlin, (2007).

(2) Nabijou S, Hobbs RE, "Frictional performance of wire and fibre ropes bent over sheaves", Journal of strain analysis for engineering design, (1995), vol. 30, 1, pp. 45-57

(3) Vennemann O, Tornqvist R, Ernst B, Winter S, Frazer I, "Bending fatigue tests using a suitable NDT method to determine lifetime of large diameter wire ropes for offshore lifting applications", Proc. OMAE (2008), Portugal, paper 57128.

(4) Davies P, Reaud Y, Dussud L, Woerther P, "Mechanical behaviour of HMPE and aramid fibre ropes for deep sea handling operations". Ocean Engineering 38 (2011), pp. 2208-2214

(5) DISH (Deep water Installation of Subsea Hardware) Joint Industry Project (2001-2006).

(6) Tornqvist R, Strande M, O'Cannell D, Gledhill P, Smeets P, Gilmore J, "Deployment of Subsea equipment: qualification of large diameter fiber rope for deepwater construction applications", OTC21588 (2011)

(7) Torben S, Ingeberg P, Bunes O, Bull S, Paterson J, Davidson D, "Fibre rope deployment system, for ultra deepwater installations", OTC18932 (2007)

(8) Thomas R, Gilmore J, "Deepwater synthetic lowering and lifting lines with enhanced cyclic bend fatigue resistance", Proc. DOT, New Orleans, (2009)

(9) Gilmore J, Stenvers D, Chou R, Some Recent Developments of Rope Technologies - Further Enhancements of High Performance Ropes, Proc. OCEANS08 (2008).

(10) Durville D, "Mechanical modeling of wire ropes in large displacements taking into account contactfriction interactions between elementary wires", E. Oñate and S. R. Idelsohn Eds., CIMNE, Barcelona, Spain, Computational Mechanics, New Trends and Applications, Jun. 29 - Jul. 2, Buenos Aires, Argentina, (1998)

(11) Durville D., "Numerical simulation of entangled materials mechanical properties" Journal of Materials Science, vol. 40, 22, pp. 5941-5948, (2005).

(12) Durville D., "Contact-friction within large assemblies of fibers for the simulation of textile structures mechanical behaviour", 7th World Congress on Computational Mechanics, 16-22/07/2006, Los Angeles, (2006). 